\title{
Vectoring for True North: Building a Research Base on Family Support
}

\author{
Kimberly Eaton Hoagwood • Barbara J. Burns
}

Published online: 5 September 2013

(C) Springer Science+Business Media New York 2013

When a parent seeks mental health services for their child, particularly if they are uninsured and poor, they are faced with systemic impediments that are often debilitating and always daunting. In addition to the scarcity of mental health specialists, parents face stigma (Hinshaw 2007), stereotypic threats (Heflinger and Hinshaw 2010), long wait lists (sometimes 6 months or more), and lack of experienced and competent providers (U.S. Department of Health and Human Services, The Substance Abuse and Mental Health Services Administration (USDHHS SAMHSA) 2013). In short, they have to deal with a fragmented, discontinuous, and non-responsive "system." Even using the word "system" to describe what families face is generous.

In 2001, The Institute of Medicine (IOM) released its report entitled, Crossing the Quality Chasm (IOM 2001). The report outlined six principles for quality care: safety, effectiveness, patient-centeredness, timeliness, efficiency, and equity. Patient-centered care was called "true north" by Berwick (2002) because it conceptualized that compass point as the ultimate destination for quality care.

The Quality Chasm report endorsed the overarching purpose proposed by the President's Advisory Commission on Consumer Protection and Quality in the Health Care Industry (1998): "The purpose of the health care system is

\section{K. E. Hoagwood ( $\square)$}

Department of Child and Adolescent Psychiatry, New York University Child Study Center, New York University School of Medicine, One Park Avenue at East 33rd, 8th Floor, New York, NY 10016, USA

e-mail: Kimberly.Hoagwood@nyumc.org

\section{B. J. Burns}

Department of Psychiatry and Behavioral Sciences, Duke University School of Medicine, Durham, NC, USA to reduce continually the burden of illness, injury, and disability, and to improve the health status and function of the people of the United States"(p. 83).

True North retains its significance as an organizing principle in healthcare and in mental health care. In the child mental health system, ensuring quality services for families with children with mental health needs is the endpoint, and the sine qua non. (We will use the term "families" to refer to parents/caregivers, and their children). Systematizing services that will improve the quality of mental health outcomes for families, however, requires a shift away from a unilateral focus on children's needs to a focus that encompasses the perspectives, needs, values, and experiences of parents, caregivers, and other significant adults. This is not a small change. It requires a different knowledge base and a new set of tools.

The research base on effective treatments and services for children has grown exponentially over the past three decades (U.S. Public Health Service 2000; U.S. Department of Health and Human Services 1999), but research on the supportive services that enable and empower families to sustain their child's treatment gains has been remarkably lacking (Hoagwood et al. 2010; Cavaleri et al. 2011).

The papers in this special issue begin to fill this hole. They are targeted at the supportive services for families that, when added to effective treatments for children, can create a package of care that is likely to improve outcomes on a broad scale. We as editors see the papers in this volume as advancing research, and consequently-we hope practice and policy about children's services-by adding important new knowledge to the research base. The papers describe in detail a set of services delivered by parents and for parents of children with mental health needs. These supportive services are more than a worthy goal or lofty ideal. They are specific targeted supports that include 
knowledge, skills, and practical assistance provided by trained professionals who are also parents or caregivers. The goal of these supportive services-often called family support services-is to assist other parents or caregivers to advocate for and actively participate in treatment planning for their children.

The studies described in these papers were deliberately positioned to align with the major shifts in healthcare delivery that are shaping mental health services in the future. Specifically, the studies address issues about three of the large changes to the healthcare system that are occurring, namely: (a) new workforce training competencies; (b) development and testing of quality indicators in the workplace; and (c) organizational supports in agencies to sustain changes and improve outcomes. In short, these three changes provide the impetus for the creation of a new system of family support services.

We will first provide a brief background to the three system problems that are being addressed through the healthcare changes: workforce shortages; fragmentation and poor quality; and disorganized organizations. Second, we outline some of the problems that healthcare reform is trying to address, changes that are redefining the contours of the mental health system. Third, we describe the contributions that the five papers in this volume are making to align with these healthcare changes.

\section{Persistent Mental Health System Inadequacies}

Workforce shortages in the children's mental health system have been documented for years (USDHHS, SAMHSA 2013; New Freedom Commission on Mental Health 2003; Hoge et al. 2005, 2007; Burns et al. 1999; Knitzer 1982). The lack of capacity to serve the needs of children and families is reflected in the persistent gap between children's mental health needs and the use of services (Merikangas et al. 2009; Kessler et al. 2005; Wang et al. 2005). In fact, the workforce capacity, such as it is, appears to be shrinking (Hoge et al. 2007; Thomas et al. 2009). There is an inadequate supply of trained social workers, psychologists, and psychiatrists, especially for the most needy populations (Hoge et al. 2007; Robiner 2006). To address workforce shortages and the high costs of health care (17.7\% of gross national product) (OECD 2013), the concept of task-shifting provides an important option, observed in medical care with the introduction of providers such as physician assistants. The World Health Organization (WHO 2008) has defined this concept as follows: "Task-shifting involves the rational redistribution of tasks among health workforce teams. Specific tasks are moved, where appropriate, from highly-qualified health workers to health workers with shorter training and fewer qualifications in order to make more efficient use of the available human resources for health" (p. 2).
A second system problem has been persistent fragmentation of the services, leading to poor quality and discontinuous care (IOM 2006). Numerous studies have documented both the inadequacy of usual care (Bickman et al. 1997; Weisz et al. 2013) and the lack of evidence to support improved outcomes for children who receive usual care. The poor quality is in part a function of an inadequately trained workforce and systemic factors, including fragmented and discontinuous service provision and use, and inconsistent incentives for agencies to provide quality services (Bickman 2013).

The third failure inheres in lack of attention to organizational context-the characteristics of work environments that are known to generate high-quality services and positive outcomes for children. Decades of studies have identified the key organizational factors that predict successful outcomes from human service agencies (Glisson et al. 2012; Olin et al. 2013). However, it is only recently that the mental health system has taken seriously the opportunity to improve the culture and climate of a service agency's work environment in order to support the installation of evidence-based practices, improve outcomes, and improve the efficiency of service delivery (Gray 2013; Hoagwood et al. 2013).

One approach to partially redress the problems of workforce shortages, poor quality, and insufficient organizational capacity has been to augment the workforce with highly-trained peer provider specialists—service providers who can deliver services directly to parents. In adult mental health, peer providers are consumers of mental health services. In contrast, in child mental health, such peer providers-often referred to now as family support specialists-are parents or caregivers who are raising or have raised a child with mental health issues. In both situations, peer providers know the service system viscerally. They have lived with the challenges of getting (or more often not getting) services. In child mental health, family support specialists are uniquely qualified to educate and empower parents to take an active role in their child's care. They are often passionate in their commitment to help other parents avoid the long and frustrating journey they have undergone (Flynn 2005).

The augmentation of the workforce with trained family support specialists is rapidly increasing; one-quarter of states now make such family support services a billable service through Medicaid or federal block grants (Center for Health Care Strategies Inc. 2012). However, with this change to the workforce comes new challenges: how to ensure that the roles, discrete functions, and competencies of the staff are clear; how to create work environments and staff units that are engaged, proficient, and effective; how to create clear communication channels within an agency; and how to create cohesive and well-functioning teams that 
reflect the family perspective and strengthen (rather than infantilize or patronize) families; in short, how to improve the overall quality of mental health services within agencies.

Quality improvement of mental health services is now poised for a major overhaul. The system problems of worker shortages, fragmentation and discontinuous services, and insufficient organizational capacity-problems that have plagued children's mental health services for decades-could be eliminated or at least drastically reduced given changes in the healthcare landscape. These changes are being driven by the repercussions from the Affordable Care Act (ACA) and concomitant healthcare financing and policies enacted by states. The changes include attention to quality of services including quality measurement, outcomes and costs, and to organizational supports (fiscal, electronic, supervisory, structural), with the goal of creating efficiencies within provider systems to better distribute and manage the diverse expertise needed to create a truly functioning "system." Related provisions include support for training professionals and reimbursement for peer professionals (such as family support specialists), opening the door to the sustainability of family support services.

\section{Healthcare Changes}

The major change effected by the Affordable Care Act is access to health insurance; it also includes provisions to make healthcare safer, more affordable, and of higher-quality (Conway et al. 2013). One essential element to the ACA is promoting reliable and meaningful quality measurement that includes outcomes related to patient experiences.

To make measurement of care practical for the new organizational shifts, measures will need to be feasible and non-burdensome. Lengthy interviews with complex and nested questions will not work. In addition, decisions about how data elements for a given measure are collected and used as part of standard clinical care will be essential. These are fundamental changes that differ in profound ways from traditional clinical measurement. These changes redirect federal and state financing streams and concomitant regulations in such a way as to reduce support for traditional separate feefor-service programs, and to incentivize support for the development of quality measures, especially those that can be used to assess quality improvement at programmatic and individual client levels.

There are numerous limitations of available sets of measures and how they are used (or more often not used). Berenson et al. (2013) describe the growth of performance measurement, and the challenge of simultaneously advancing quality measurement while avoiding the potential for unintended adverse consequences. For instance, the scope of available measures has limited the potential usefulness of some measurement efforts, particularly for consumers. Public reporting and pay-for-performance is growing (Ryan et al. 2012).

Berenson et al. (2013) recommend using quality measures strategically and adopting other quality improvement approaches where measures fall short. They also recommend measuring quality at the level of the organization or program, not solely at the level of the provider. Conway et al. (2013) point out that limited measures exist in some domains (e.g., care coordination and patient engagement), and more program and system-based outcome measures with high public health relevance are needed.

What is important here is that the changes in the healthcare system are driving services away from individual models of care delivery-such as solo, fee-for-service practices and complex psychological assessments of intrapsychic functioning - towards team-based and regionalized networks of providers, electronically-shared client data, and the use of brief metrics to assess outcomes of services at organizational (agency), provider, and client levels. The workforce will need to be expanded and trained to deal with the increased demands (volume) of persons seeking care and to provide the essential supportive services beyond specific treatments (medication or therapeutic) that are needed to sustain positive outcomes. Consequently, the workforce will need to include trained and certified peer specialists. In the children's system these individuals tend to be called family support specialists.

\section{The Papers in this Volume}

The articles in this special issue describe results from a National Institute of Mental Health funded study (R01MH085969) of family support services provided to parents of children and adolescents with serious mental health issues by family support specialists-i.e., parents themselves who have taken a professional position in agencies in order to deliver services directly to parents. The study is the first in the children's mental health field to describe family support specialists' roles, functions, and especially their work as members of a team that includes other professionals. This study describes aspects of the organizational context of their work environment that are likely to affect team functioning, quality of the program, as well as the quality of the individual family support specialist's work.

\section{The Five Papers}

The first paper (Developing Quality Indicators for Family Support Services in Community Team-Based Mental 
Health Care) describes a consensus process among a group of child and family mental health experts to develop quality indicators for family support services. Because quality metrics are gaining ascendancy within health care as a means of promoting safe, effective, timely, efficient, equitable and patient-centered health care (IOM 2001), they have become an essential element of system accountability. Developing these indicators for family support will reinforce this service model and align it with other areas of healthcare. Thus, this paper makes a very important contribution.

Unlike other areas of health where clear models for effective interventions exist, for parent-delivered family support, the data are suggestive but not definitive. It could be argued that developing quality indicators is thus premature. We disagree. We believe the indicators developed through the process described in this paper create concordance between this service model and the new healthcare emphasis on accountability, competencies, and performance standards.

The second paper (What Family Support Specialists Do: Examining Service Delivery) provides an analysis of qualitative interviews, observational data and quantitative survey data from 63 staff members at 21 mental health programs in New York State. One goal was to assess the similarities and differences between family support specialists and other staff about their perceptions of these services, and to assess whether these perceptions of activities are congruent with what the specialists actually did. This paper advances the field by documenting carefully the types of actual services provided by family support specialists, as well as whether those activities are recognized by the other members of the program staff with whom they work. Implications of these findings are discussed in the context of developing competencies and of the quality indicators described in the first paper.

The third paper (The Organizational Social Context (OSC) of Mental Health Medicaid Waiver Programs with Family Support Services: Implications for Research and Practice) describes the organizational cultures and climates of the programs that employed the family support specialists. It assesses variation in job satisfaction and organizational commitment for the specialists $(N=37)$ and other staff $(N=172)$ who were largely caseworkers, as a function of program culture and climate. This is the first study of its kind to use a well-established organizational assessment (the OSC) to profile programs that employ family support specialists. Latent profile analysis (LPA) classified the programs, and hierarchical linear models (HLM) analysis assessed the association of program-level culture and climate with individual staff-level job satisfaction and commitment. The analysis indicated that family support specialists and other staff did not differ in job satisfaction, and that organizational commitment was higher for both groups in programs with positive cultures and climates. This is important because it suggests that the OSC is a useful measure in these kinds of programs, and can yield information about the kinds of malleable context variables that can be changed to improve family and child outcomes.

The fourth paper (Quality Indicators for Family Support Services and Their Relationship to Organizational Social Context) explores whether performance on quality benchmarks targeting two levels of indicators (program and individual family support specialist practices) relate to the organizational social context profiles described in the paper above. Understanding how specific culture and climate dimensions relate to the delivery of family support services was important in our study because quality improvement of programs depends upon the identification of modifiable dimensions of context and strategies to improve the integration of these specialists within these programs. These are the goals of Phase 2 of the study, which is currently in the field.

This paper describes some of the distinct differences that emerged for staff-level quality indicators. "Best" program profiles were associated with higher-quality individual family support specialist practices, while "worst" program profiles were linked with poorer quality specialist practices, particularly with inappropriate practices. This paper also provides some support for the concurrent validity of the quality indicators described earlier. The availability of empirically-derived and consensually-supported quality metrics is a major step forward in strengthening the knowledge base and practical applicability of family support services.

The fifth paper (Quality Indicators for Multidisciplinary Team Functioning in Community-Based Children's Mental Health Services) examines the relationship of OSC profile variables in relationship to five quality indicators of family support programs related specifically to team functioning. Team functioning was selected as a construct because the ability to maximize the unique competencies and skills of family support specialists is likely to depend upon the clarity of roles, the cohesion of teams, and their functioning. Consonant with the findings from the other papers in this series, the authors found that programs with higher performance on structures to facilitate teamwork, informal communication mechanisms among team members, and the ability to integrate family support specialists into the team as equal partners displayed more positive organizational functioning. Importantly, however, on one of the indicators deemed to be especially important in states implementing family support-i.e., specialized supervision for the specialists-overall performance on this indicator was very low. Over three-quarters of programs fell below 
performance expectations. Thus, the quality indicators can be used to pinpoint problem areas, as well as to identify work-related dimensions that are functioning well.

\section{Conclusion}

The five papers in this issue build on the rich and systematic development of family support services research by a team that represented the perspectives of policy-makers, practitioners, advocates, and scientists. The prior work of this group set the foundation for the current work by creating a theoretical and conceptual framework, followed by the design and testing of a training curriculum for family support specialists implemented across all of New York State (Olin et al. 2010a, b; Rodriguez et al. 2011; Wisdom et al. 2011). However, despite intensive training and consultation, these early efforts uncovered significant difficulties in integrating family support specialists into the workforce, particularly given the systemic and organizational impediments of poorly-coordinated and inadequate care. The five papers therefore focus on the development of tools, strategies, and approaches to improve the quality of this service model, including advances in quality measurement, team functioning, and the impact of organizational culture and climate. The cumulative sequencing of the services research agenda that undergirds this work offers a model for other service programs, and provides a vehicle for moving family support services forward in the context of both deficiencies in the child and family service system and opportunities for higher-quality care under the ACA.

As we vector towards true north in mental healthcarewith family-centered care as the locus and guiding principle-family support services will take on new value. The papers in this special issue advance knowledge about this service model by providing new quality improvement tools to enable further testing of the effectiveness of family support services and their disseminability through specific attention to organizational context.

Acknowledgments The authors would like to thank all family advisors in New York State and nationally, including Frances S. Purdy, MEd, JD, Darcy Gruttadaro, JD, Elaine Slaton, and Trina Osher, PhD. We also thank the New York State Office of Mental Health, including former commissioner Michael Hogan, former Acting Commissioner Kristin Riley, and Deputy Commissioner Donna Bradbury for state leadership on family support. This research was supported by grants from the National Institute of Mental Health (NIMH) (Grants R01MH085969 and P30MH090322).

\section{References}

Berenson, R. A., Pronovost, P. J. \& Krumholz, H. M. (2013). Achieving the Potential of Health Care Performance Measures.
Washington, DC: The Urban Institute. Retrieved July 30, 2013, from http://www.urban.org/health_policy/url.cfm?ID=412823.

Berwick, D. M. (2002). A user's manual for the IOM's "Quality Chasm" report. Health Affairs, 21(3), 80-90. doi:10.1377/ hlthaff.21.3.80.

Bickman, L. (2013). Facing reality and jumping the chasm. Administration and Policy in Mental Health and Mental Health Services Research, 40(1), 1-5. doi:10.1007/s10488-012-0460-6.

Bickman, L., Sumerfelt, W. T., \& Noser, K. (1997). Comparative outcomes of emotionally disturbed children and adolescents in a system of services and usual care. Psychiatric Services, 48, $1543-1548$.

Burns, B. J., Hoagwood, K., \& Mrazek, P. (1999). Effective treatment for mental disorders in children and adolescents. Clinical Child and Family Psychology Review, 2, 199-254.

Cavaleri, M. A., Olin, S. S., Kim, A., Hoagwood, K. E., \& Burns, B. J. (2011). Family support in prevention programs for children at risk for emotional/behavioral problems. Clinical Child and Family Psychology Review, 14(4), 399-412.

Center for Health Care Strategies Inc. (2012). Medicaid Financing for Family and Youth Peer Support: A Scan of State Programs. Hamilton, NJ: The Center for Health Care Strategies. Retrieved July 30, 2013, from http://www.chcs.org/publications3960/ publications_show.htm?doc_id=1261373.

Conway, P. H., Mostashari, F., \& Clancy, C. (2013). The future of quality measurement for improvement and accountability. Journal of the American Medical Association, 309(21), 2215-2216. doi:10.1001/jama.2013.4929.

Flynn, M. (2005). Family perspectives on evidence-based practice. Child and Adolescent Psychiatric Clinics of North America, 14(2), 217-224.

Glisson, C., Hemmelgarn, A., Green, P., Dukes, D., Atkinson, S., \& Williams, N. (2012). Randomized trial of the ARC organizational intervention with community-based mental health programs and clinicians serving youth. Journal of the American Academy of Child and Adolescent Psychiatry, 51(8), 780-787.

Gray, J. A. (2013). The shift to personalised and population medicine. Lancet, 382(9888), 200-201. PubMed PMID: 23878859.

Heflinger, C. A., \& Hinshaw, S. P. (2010). Stigma in child and adolescent mental health services research: understanding professional and institutional stigmatization of youth with mental health problems and their families. Administration and Policy in Mental Health, 37, 61-70.

Hinshaw, S. P. (2007). The mark of shame: stigma of mental illness and an agenda for change. New York: Oxford University Press.

Hoagwood, K. E., Cavaleri, M. A., Olin, S., Burns, B. J., Slaton, E., Gruttadaro, D., et al. (2010). Family support in children's mental health: a review and synthesis. Clinical Child and Family Psychology Review, 13(1), 1-45.

Hoagwood, K., Olin, S., \& Cleek, A. (2013). Beyond context to the skyline: thinking in 3D. Administration and Policy in Mental Health, 40(1), 23-28.

Hoge, M. A., Morris, J. A., Daniels, A. S., Huey, L. Y., Stuart, G. W., Adams, N., et al. (2005). Report of recommendations: the Annapolis Coalition Conference on Behavioral Health Work Force Competencies. Administration and Policy in Mental Health, 32(5-6), 651-663.

Hoge, M.A., Morris, J.A., Daniels, A.S., Stuart, G.W., Huey, L.Y., and Adams, N. (2007). An action plan on behavioral health workforce development. Cincinnati, Ohio: The Annapolis Coalition on the Behavioral Health. Retrieved July 30, 2013, from: www.annapoliscoalition.org or at http://www.samhsa.gov/matrix $2 /$ matrix_workforce.aspx.

Institute of Medicine. (2001). Crossing the quality chasm: a new health system for the 21st century. Washington, DC: National Academies Press. 
Institute of Medicine. (2006). Improving the quality of health care for mental and substance-use conditions. Washington, DC: National Academies Press.

Kessler, R. C., Berglund, P., Demler, O., Jin, R., Merikangas, K. R., \& Walters, E. E. (2005). Lifetime prevalence and age-of-onset distributions of DSM-IV disorders in the National Comorbidity Survey Replication. Archives of General Psychiatry, 62(6), 593-602. doi:10.1001/archpsyc.62.6.593.

Knitzer, J. (1982). Unclaimed children: the failure of public responsibility to children and adolescents in need of mental health services. Washington: Children's Defense Fund.

Merikangas, K. R., Nakamura, E. F., \& Kessler, R. C. (2009). Epidemiology of mental disorders in children and adolescents. Dialogues in Clinical Neuroscience, 11(1), 7-20.

New Freedom Commission on Mental Health (2003). Achieving the Promise: Transforming Mental Health Care in America. Final Report. DHHS Pub. No. SMA-03-3832. Rockville, MD: Substance Abuse and Mental Health Administration.

Olin, S. S., Hoagwood, K. E., Rodriguez, J., Radigan, M., Burton, G., Cavaleri, M., et al. (2010a). Impact of empowerment training on the professional work of family peer advocates. Children and Youth Services Review, 32(10), 1426-1429.

Olin, S. S., Hoagwood, K. E., Rodriguez, J., Ramos, B., Burton, G., Penn, M., et al. (2010b). The application of behavior change theory to family-based services: improving parent empowerment in children's mental health. Journal of Child and Family Studies, 19(4), 462-470.

Olin, S. S., Williams, N., Pollock, M., Armusewicz, K., Kutash, K., Glisson, C., \& Hoagwood, K. E. (2013). Quality indicators for family support services and their relationship to organizational social context. Administration and Policy in Mental Health and Mental Health Services Research. Published online May 25, 1-12. doi:10.1007/s10488-013-0499-z.

Organisation for Economic Co-operation and Development (2013). Organisation for Economic Co-operation and Development (OECD) Health Data 2013. Paris, France: OECD. Retrieved July 30, 2013, from http://www.oecd.org/health/healthdata.

President's Advisory Commission on Consumer Protection and Quality in the Health Care Industry (1998). Quality First: Better Health Care for All Americans. Rockville, MD: The Agency for Healthcare Research and Quality.

Robiner, W. N. (2006). The mental health professions: workforce supply and demand, issues, and challenges. Clinical Psychology Review, 26, 600-625.

Rodriguez, J., Olin, S. S., Hoagwood, K. E., Shen, S., Burton, G., Radigan, M., et al. (2011). The development and evaluation of a parent empowerment program for family peer advocates. Journal of Child and Family Studies, 20(4), 397-405.
Ryan, A. M., Nallamouth, B. K., \& Dimik, J. B. (2012). Medicare's public reporting initiative on hospital quality had modest or no impact on mortality from three key conditions. Health Affairs, 31(5), 585-592.

Thomas, K. C., Ellis, A. R., Konrad, T. R., Holzer, C. E., \& Morrissey, J. P. (2009). County-level estimates of mental health professional shortage in the United States. Psychiatric Services, 60(10), 1323-1328. doi:10.1176/appi.ps.60.10.1323.

U.S. Department of Health and Human Services. (1999). Mental Health: A Report of the Surgeon General. Rockville: U.S. Department of Health and Human Services, Substance Abuse and Mental Health Services Administration, Center for Mental Health Services, National Institutes of Health, National Institute of Mental Health.

U.S. Department of Health and Human Services, Substance Abuse and Mental Health Services Administration (USDHHS, SAMHSA)(2013). Report to Congress on the Nation's Substance Abuse and Mental Health Workforce Issues. January 24, 2013. Rockville, MD: Substance Abuse and Mental Health Services Administration. Retrieved from http://store.samhsa.gov/product/ Report-to-Congress-on-the-Nation-s-Substance-Abuse-and-MentalHealth-Workforce-Issues/PEP13-RTC-BHWORK.

U.S. Public Health Service. (2000). Report of the Surgeon General's Conference on Children's Mental Health: A National Action Agenda. Washington, DC: Department of Health and Human Services.

Wang, P. S., Lane, M., Olfson, M., Pincus, H. A., Wells, K. B., \& Kessler, R. C. (2005). Twelve-month use of mental health services in the United States: results from the National Comorbidity Survey Replication. Archives of General Psychiatry, 62(6), 629-640.

Weisz, J. R., Kuppens, S., Eckshtain, D., Ugueto, A. M., Hawley, K. M., \& Jensen-Doss, A. (2013). Performance of evidence-based youth psychotherapies compared with usual clinical care: a multilevel meta-analysis. Journal of American Medical Association Psychiatry, 70(7), 750-761. doi:10.1001/jamapsychiatry. 2013.1176.

Wisdom, J. P., Olin, S., Shorter, P., Burton, G., \& Hoagwood, K. E. (2011). Family peer advocates: a pilot study of the content and process of service provision. Journal of Child and Family Studies, 20(6), 833-843.

World Health Organization (2008). Task shifting: Rational redistribution of tasks among health workforce teams: Global recommendations and guidelines. Geneva: World Health Organization. Retrieved on July 30, 2013, from: http://www.who.int/health systems/TTR-TaskShifting.pdf. 